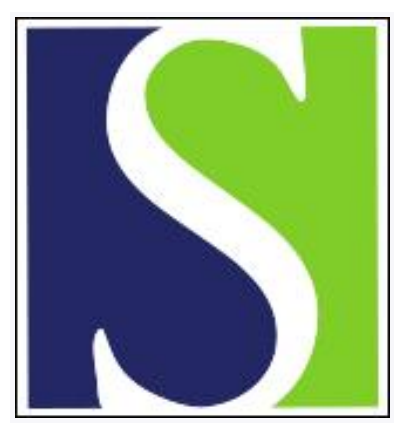

Scand J Work Environ Health 2001;27(2):120-124

https://doi.org/10.5271/sjweh.598

Issue date: Apr 2001

Chromosome aberrations in lymphocytes of persons exposed to an earthquake in Armenia

by Nersesyan AK, Boffetta P, Sarkisyan TF, Zalinyan GG, Arutyunyan RM

Affiliation: State University, Yerevan, Armenia.

Key terms: Armenia; chromosome aberration; earthquake; exposure; lymphocyte; psychogenic stress

This article in PubMed: www.ncbi.nlm.nih.gov/pubmed/11409594

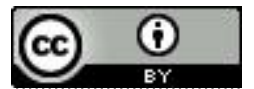




\title{
Chromosome aberrations in lymphocytes of persons exposed to an earthquake in Armenia
}

\author{
by Armen K Nersesyan, ${ }^{1,3}$ Paolo Boffetta, ${ }^{2}$ Tamara F Sarkisyan, ${ }^{1}$ Gayane G Zalinyan, ${ }^{1}$ \\ Rouben M Arutyunyan ${ }^{1}$
}

\begin{abstract}
Nersesyan AK, Boffetta P, Sarkisyan TF, Zalinyan GG, Arutyunyan RM. Chromosome aberrations in lymphocytes of persons exposed to an earthquake in Armenia. Scand J Work Environ Health 2001;27(2):120-124.

Objective This study attempted to determine the level of chromosome aberrations in lymphocytes of victims of the 1988 earthquake in Armenia.

Methods Chromosome aberrations were measured in blood samples taken from 41 victims of the earthquake that hit Armenia in 1988 and in samples of 47 reference blood donors. The victims suffered from severe psychoemotional stress but were otherwise healthy. All the samples were taken 2 to 3 weeks after the earthquake. All the subjects were lifetime nonsmokers. The cells were scored blind as to the exposure status.

Results The subjects exposed to the earthquake had a higher proportion of cells with chromosome aberrations [3.1 (SD 2.1)\%] than the referents [1.7 (SD 1.3)\%, P-value for the difference 0.0009]. The difference persisted when the values were adjusted for age and gender [relative risk (RR) 1.9, 95\% confidence interval $(95 \% \mathrm{CI})$ 1.4-2.5]. The difference was present for double breaks (RR 4.1, 95\% CI 2.6-6.4), but not for single breaks (RR 1.1, 95\% CI 0.8-1.7). The exposed subjects also had a lower percentage of cells with 46 chromosomes $(\mathrm{P}=0.03)$ than the referents.

Conclusions This study suggests an increase in chromosome aberrations in the lymphocytes of victims of a severe earthquake as compared with the levels of referents. If not due to bias or confounding, the difference may reflect the effect of either environmental exposures related to the earthquake or severe psychogenic stress. The levels of chromosome aberrations found among the earthquake victims in this study are comparable with those found in prospective studies of long-term cancer risk.
\end{abstract}

Key terms psychogenic stress.

Wild rodents living in seismic active regions have an increased level of chromosome aberrations (CA) and chromosome instability in somatic cells (1). An earthquake was shown to act as an immunodepressive agent on (C57B1/6xCBA)F1 mice in Tajikistan (2). It is unclear whether this effect, if real, can be explained by psychogenic stress linked to the earthquake or by chemical and physical characteristics of the seismic regions. In favor of the first interpretation, a study has reported that psychogenic stress increases chromosome aberrations in rats (3). In favor of the second interpretation, a study carried out in 1987 , of the spontaneous mutation level in bone marrow cells of rodents from Armenian regions prone to expected severe earthquakes, also showed an increased level of chromosome aberrations (4).

To our knowledge, the possible influence of an earthquake on the level of chromosome aberrations in humans has not been studied previously. We aimed at studying the level of chromosome aberrations in lymphocytes of victims of the 1988 earthquake in Armenia.

1 State University, Yerevan, Armenia.

2 International Agency for Research on Cancer, Lyon, France.

3 Cancer Research Centre, Yerevan, Armenia.

Correspondence to: Dr Paolo Boffetta, Chief, Unit of Environmental Cancer Epidemiology, International Agency for Research on Cancer (IARC), 150 cours Albert-Thomas, 69008 Lyon, France. [E-mail: boffetta@iarc.fr] 


\section{Subjects and methods}

A devastating earthquake occurred in Armenia on 7 December 1988; it affected $40 \%$ of the entire territory of the country. More than 25000 people died, 32500 were wounded, and 530000 lost their homes.

Two to three weeks after the disaster, blood samples were collected from 41 earthquake victims from Leninakan (now Giumry). All the participants were volunteers and had no physical damage or other health problems, except severe psychoemotional stress. Ten of the subjects lost all members of their family, 18 lost at least 1 family member, and 8 subjects, at the time of the blood collection, had no information concerning the fate of their relatives. As referents we used 47 healthy volunteers from Yerevan who donated blood to the earthquake victims. All the subjects included in this study were lifetime nonsmokers; data on smoking have been gathered via a personal questionnaire.

Chromosome specimens were prepared according to a previously described protocol (5). All the blood samples were processed by the same technicians under identical conditions. Blood was obtained by venipuncture from the study participants and collected in tubes with $50 \mathrm{IU} / \mathrm{ml}$ of heparin. Immediately after the collection, the blood samples were cultured and harvested using standard procedures (6). After 74 hours of incubation, colchicine $(0.25 \mu \mathrm{g} / \mathrm{ml})$ was added to the cultures. After 2 hours, the cells were harvested, and the supernatant was resuspended in a $0.55 \%$ potassium chloride solution and fixed in methanol-acetic acid (3:1). The slides were prepared after the harvesting by the air-drying technique. They were stained with Giemsa stain. Only cells with $45-47$ centromeres were analyzed. The percentage of cells with aberrations, of either the chromatid or chromosome type (single and double breaks, respectively), and exchanges was calculated, and the total number of structural aberrations was scored. Gaps were considered separately. At least 100 cells were analyzed for each person. The slides were read and scored blindly as to exposure status.

We compared the chromosome aberration scores in the 2 groups using the t-test and nonparametric (Wilcoxon and Kruskal-Wallis) tests. The analyses were repeated after log transformation of the chromosome aberration scores. In addition, we conducted a multivariate Poisson regression analysis, using the level of total chromosome aberrations, the level of single and double breaks, and the proportion of cells with alterations as dependent variables (7). Age, gender, and exposure to the earthquake were the explanatory variables. Age was added to the models as a continuous variable and also in 4 categories. Since this choice did not influence the results for the other variables, we have presented the results of the models including the categorical variable.

\section{Results}

The selected characteristics of the study population are presented in table 1 . A total of 51 women and 37 men was studied. Their mean age was 37.7 (range 10-71, SD 14.2) years.

The mean total aberrant cell score was 2.4 (standard deviation 1.9) for the total population. The mean score of aberrant cells was higher in the earthquake-exposed group than in the reference group (table 2). The P-value of the t-test for the difference between the means was 0.0002 ; that of the Wilcoxon test was 0.0009 . The results of the Poisson regression analysis confirmed the presence of a difference (table 3). The relative risk (RR) for exposure was 1.9 [95\% confidence interval $(95 \% \mathrm{CI})$ $1.4-2.5]$.

The average level of single chromosome breaks was 1.3 (SD 1.4); it was similar for the exposed subjects and the referents (table 2). The lack of a difference was confirmed in the multivariate Poisson regression analysis (RR 1.1, 95\% CI 0.8-1.7). With respect to double breaks, the average level was 1.3 (SD 1.4), and it was higher for the exposed subjects than the referents (table $2)$. The relative risk was 4.1 (95\% CI 2.6-6.4). The number of chromosome aberrations per studied cell was higher in the exposed subjects [3.3 (SD 0.29)\%] than in the referents $[1.9 \%$ (SD 0.15$) \%]$. The relative risk was $2.1(95 \%$ CI 1.6-2.8).

After adjustment for the other variables, there was a small, nonsignificant increase in the chromosome

Table 1. Age and gender distribution of the subjects by exposure to an earthquake.

\begin{tabular}{lccc}
\hline Group & Exposed subjects & Referents & Total \\
\hline Age & & & \\
$10-25$ years & 10 & 8 & 18 \\
26-34 years & 9 & 9 & 26 \\
35-49 years & 12 & 13 & 21 \\
50-71 years & 10 & & 23 \\
Gender & & 22 & 37 \\
$\quad$ Male & 15 & 25 & 51 \\
$\quad$ Female & 26 & 47 & 88 \\
\hline Total & 41 & & \\
\hline
\end{tabular}

Table 2. Results of the univariate analysis with exposure to the earthquake and level of chromosome aberrations (\% of cells).

\begin{tabular}{|c|c|c|c|c|c|c|}
\hline \multirow[b]{2}{*}{ Group } & \multicolumn{2}{|c|}{ All aberrations } & \multicolumn{3}{|c|}{ Single breaks } & Double breaks \\
\hline & Mean SD & Range & Mean & SD & Range & Mean SD Range \\
\hline Exposed & 3.12 .1 & $0-8$ & 1.3 & 1.8 & $0-8$ & $2.2 \quad 0.50-5.5$ \\
\hline Reference & 1.71 .3 & $0-7$ & 1.2 & 1.0 & $0-5$ & $0.5 \quad 0.6 \quad 0-2$ \\
\hline -value ${ }^{\mathrm{a}}$ & 0.0009 & & & 0.3 & & $<0.0001$ \\
\hline
\end{tabular}

a Wilcoxon rank-sum test.

Scand J Work Environ Health 2001, vol 27, no 2 
Table 3. Results of the Poisson regression analysis. (RR = relative risk, adjusted for the variables in the table, $95 \% \mathrm{Cl}=$ confidence interval)

\begin{tabular}{|c|c|c|c|c|c|c|}
\hline & \multicolumn{2}{|c|}{ All aberrations } & \multicolumn{2}{|c|}{ Singe breaks } & \multicolumn{2}{|c|}{ Double breaks } \\
\hline & RR & $95 \% \mathrm{Cl}$ & $\mathrm{RR}$ & $95 \% \mathrm{Cl}$ & $\mathrm{RR}$ & $95 \% \mathrm{Cl}$ \\
\hline Female gender ${ }^{\mathrm{a}}$ & 0.7 & $0.5-1.0$ & 0.6 & $0.4-0.8$ & 1.0 & $0.7-1.5$ \\
\hline \multicolumn{7}{|l|}{$\mathrm{Age}^{\mathrm{b}}$} \\
\hline $26-34$ years & 0.9 & $0.6-1.4$ & 0.8 & $0.4-1.3$ & 1.1 & $0.6-1.9$ \\
\hline $35-49$ years & 1.1 & $0.7-1.6$ & 0.8 & $0.4-1.4$ & 1.4 & $0.8-2.3$ \\
\hline $50-71$ years & 1.0 & $0.7-1.5$ & 0.9 & $0.6-1.5$ & 1.3 & $0.7-2.2$ \\
\hline $\begin{array}{l}\text { Exposure to } \\
\text { earthquake }\end{array}$ & 1.9 & $1.4-2.5$ & 1.1 & $0.8-1.7$ & 4.1 & $2.6-6.4$ \\
\hline
\end{tabular}

a Reference category: male gender.

${ }^{\mathrm{b}}$ Reference category: age $<26$ years.

c Reference category: unexposed to earthquake (reference group)

aberration level with increasing age. The women had a lower aberrant cell score (mean 2.1, SD 1.8) than the men (mean 2.7, SD 1.9). This difference was confirmed in the Poisson regression analysis (RR $0.7,95 \% \mathrm{CI}$ $0.5-1.0)$.

When we restricted the analysis to the women, the results, shown in table 2 , were similar and, therefore, confirmed that gender did not play a confounding role. The relative risk for exposure to the earthquake was 2.5 (95\% CI 1.6-3.8).

The mean number of gaps was 1.1 per 100 cells among the exposed and 1.0 per 100 cells among the referents $(\mathrm{P}$-value of the difference $=0.5$ ).

\section{Discussion}

The main result of our study was a higher level of chromosome aberrations in the white blood cells of persons exposed to the earthquake in Armenia in 1988 as compared with the levels of referents. After control for potential confounders, the exposed subjects had $90 \%$ higher levels of chromosome aberrations than the referents. The difference was due to a higher level of double breaks among the exposed subjects. No difference was found in the proportion of cells with gaps. When these results are interpreted, it is important to consider several possible sources of bias.

Several environmental exposures are known to induce chromosome aberrations in humans. We were able to control for smoking since all our subjects were lifelong nonsmokers. It is unlikely that exposure to other known clastogens, such as chemotherapy agents and occupational carcinogens, explains our findings, which held also when the analysis was restricted to women. We did not collect information on diet at the time of the blood collection. However, it is unlikely that diet played a role. The diets in Yerevan and Leninakan were similar before the earthquake (8); during the first week after the earthquake only local food was available. The same questionnaire was applied to the exposed and reference subjects to assess their smoking history to reduce the likelihood of confounding by smoking. Only lifelong never smokers were retained in the study.

Blood was obtained 2 to 3 weeks after the earthquake, while access to food was reduced only during the first days after the disaster. It is possible that volunteers moving to the earthquake area, who comprised the reference group, were healthier than the Armenian population at large. The difference we observed would therefore be due to a lower chromosome aberration level in the reference group rather than to a higher level in the exposed subjects. We have no blood samples from the exposed subjects before the earthquake; however, a survey conducted during 1975-1983 among Armenian subjects not exposed to known clastogens revealed a chromosome aberration level that was comparable with the level found among the referents in this study (9). This result speaks against a strong health selection for the referents when they volunteered to help the earthquake victims.

Differential reading of the slides is an unlikely explanation for our findings, since the reading was done blind as to the exposure status. There were no differences in the processing of the blood samples or the cultivation of the leukocytes between the exposed and reference subjects. All the slides were scored by 3 experts without knowledge of the source. The interrater agreement was not formally assessed. However, slides with extreme results (ie, 0,7 or 8 chromosome aberrations) were rescored by a second expert, and the results were confirmed. Similarly, good agreement was found in the rescoring by the same experts of slides used in another study (10).

If the difference in the chromosome aberration levels between the exposed and reference subjects is real, it may have various explanations. Russian investigators had proposed that the increased level of chromosome aberrations in somatic cells of rodents trapped in regions with high seismic activity is due to an increased level of radiation from the contamination of water by radon and salts of heavy metals $(1,3)$. However, the survey on spontaneous chromosome aberration in Armenian people conducted in 1975-1983 showed similar levels in the various regions of the country, including Leninakan (9). These levels were similar to those found in our reference group. Environmental exposure to radiation or carcinogenic compounds may have been enhanced by the earthquake itself.

An alternative explanation is psychogenic stress. All the earthquake victims suffered from severe psychoemotional stress. Russian investigators have shown an 
increased level of genetic instability, including chromosome aberrations, in cells of stressed animals $(11,12)$. In addition, stress can significantly increase the activity of chemical mutagens in rodents (12). The possible reasons for the mutagenic activity of stress are a genotoxic action of endogenous factors $(13,14)$ and an increased level of lipid peroxidation $(15,16)$. The latter explanation is supported by data from Armenia showing an increased level of lipid peroxidation in victims of the same earthquake (17).

An increased level of chromosome aberrations has been shown to be associated with an increased risk of cancer in 2 cohort studies from the Nordic countries and Italy (18-20). These cohorts included readings of chromosome aberrations from several laboratories, and the definition of an elevated level of chromosome aberrations varied according to laboratory. However, a score of 3.1 for cells with chromosome aberrations, the mean value of the exposed subjects in this study, would be included in the "high" category of all the laboratories of the Nordic study, and an aberrant cell score of 1.7, the mean value of the referents in this study, would be included in the "intermediate" category in most of the laboratories (18). In other words, the magnitude of the difference found in this study between the exposed and reference subjects is comparable with that reported to predict an increased cancer risk in prospective studies. It is unclear, however, whether the increase in chromosome aberrations among the exposed subjects in our study is transient or represents a long-term phenomenon that may increase cancer risk.

This is the first study to assess the chromosome aberration levels of victims of an earthquake. The results suggest an increased level of chromosome aberrations in this group of people. Our results may be due to spurious factors and require confirmation. If real, they would not completely distinguish between a clastogenic effect of the experience of the earthquake, possibly mediated by psychogenic stress, and an effect of environmental conditions either present in seismic areas or released after the earthquake itself. The increase in the frequency of double breaks, in particular, speaks in favor of a possible role of radon or other sources of ionizing radiation.

No evidence is available for an increased risk of cancer following exposure to an earthquake, and the available evidence of an increased risk of cancer following severe psychogenic stress is inconsistent $(21,22)$. Further studies might clarify whether an increase in chromosome aberrations occurs after exposure to an earthquake or other sources of severe psychoemotional stress. Furthermore, such studies would help to clarify whether our results are due either to bias or to confounding by tobacco smoking, diet, or other factors. They would also contribute to clarifying whether such an increase, if real, is transient and insufficient to represent a carcinogenic hazard or, conversely, whether it may contribute to an increase in the long-term risk of cancer of exposed persons.

\section{Acknowledgments}

We wish to thank Lars Hagmar and Stefano Bonassi for their useful comments on the draft manuscript.

\section{References}

1. Vorontsov NN, Liapunova EA. Chromosome instability in rodents trapped in seismic active regions. Dokl Akad Nauk SSSR 1984;277:214-8.

2. Chugunov A. Earthquake depresses the immunity. Khim Zhizn 1990;5:30-1.

3. Fischman HK, Pero RW Kelly DD. Psychogenic stress induces chromosomal and DNA damage. Int J Neurosci 1996; 84:219-27.

4. Liapunova EA, Akhverdian MR, Vorontsov NN. Chromosomal instability in rodents living in mountains of Caucasus. Dokl Akad Nauk SSSR 1987;298:480-3.

5. Arutyunyan RM, Sarkisyan TF, Oganesyan GG, Durnev AD. Comparative investigation of anticlastogenic effects in cell cultures of healthy donors and patients with nettle-rash. Mutat Res 1994;320:335-41.

6. Hungerford DA. Leukocytes cultured small inocula of whole blood and the preparation of metaphase chromosomes by treatment with hypotonic KCl. Stain Technol 1965;40:333-8.

7. Bonassi S, Ceppi M, Fontana V, Merlo F. Multiple regression analysis of cytogenetic human data. Mutat Res 1994; 313:69-80.

8. Nersesyan AK, Arutyunyan RM, Groger A, Kaiser HE. Spreading of carcinogens and mutagens in the environment of Armenia and possible ways of cancer prevention. Arch Oncol. In press.

9. Arutyunyan RM, Kochar NG. Anthropology of Armenian people and ecological genetics. Yerevan (Armenia): University Press, 1996.

10. Movsesian ES, Sarkisian TF, Arutiunian RM. The cytogenetic effect of natural modifiers of mutagenesis in a human lymphocyte culture: the modification of the mutagenic effect by recombinant interferon in vitro in the lymphocytes of bronchial asthma patients and of healthy donors. Tsitol Genet 1990;24:21-4.

11. Borodin PM, Beliaev DK. Effect of stress on cross-over frequency of the 2nd chromosome of house mice. Dokl Akad Nauk SSSR 1980;253:727-9.

12. Bikovskaia NV, Diuzhikova NA, Vaido AI, Lopatina NG, Shvartsman P. Frequency of chromosome aberrations induced by stress and cyclophosphane in bone marrow cells of rats selected for the threshold of nervous system excitability. Genetika 1994;30:1224-8.

13. Borodin PM. Stress and genetic variability. Genetika 1987; 23:1003-10.

14. Kerkis J. Some problems of spontaneous and induced muta- 
genesis in mammals and man. Mutat Res 1975;29:271-7.

15. Vail'ev VK, Meerson FZ. DNA damage and reparative synthesis in various organs of the rat, induced by emotionalpainful stress. Vopr Med Khim 1984;30:112-4.

16. Guliaeva NV, Dupin AM, Levshina IP, Obidin AB, Boldyrev AA. Carnosine prevents the activation of free-radical lipid oxidation during stress. Biull Eksp Biol Med 1989;107:1447.

17. Azatyan AB, Agajanov MI, Pogosyan R. Conditions of lipid peroxidation in erythrocytes' membrane and some values of nitrogen exchanges at Apitak earthquake victims with and without crush syndrome. In: Gabrielyan ES, editor. Medical and biological problems of stress. Yerevan: Armenian National Academy of Sciences, 1997:40-1.

18. Hagmar L, Brogger A, Hansteen IL, Heim S, Hogstedt B, Knudsen L, et al. Cancer risk in humans predicted by increased levels of chromosomal aberrations in lymphocytes: Nordic Study Group on the Health Risk of Chromosome Damage. Cancer Res 1994;54:2919-22.
19. Bonassi S, Abbondandolo A, Camurri L, Dal Prá L, De Ferrari M, Degrassi F, et al. Are chromosome aberrations in circulating lymphocytes predictive of future cancer onset in humans? Preliminary results of an Italian cohort study. Cancer Genet Cytogenet 1995;79:133-5.

20. Hagmar L, Bonassi S, Stromberg U, Brogger A, Knudsen LE, Norppa H, et al. Chromosomal aberrations in lymphocytes predict human cancer: a report from the European study group on cytogenetic biomarkers and health $(\mathrm{ESCH})$. Cancer Res 1998;58:4117-21.

21. Penninx BW, Guralnik JM, Pahor M, Ferrucci L, Cerhan JR, Wallace R, Havlik RJ. Chronically depressed mood and cancer risk in older persons. JNCI 1998;90:1888—93.

22. Johansen C, Olsen JH. Psychological stress, cancer incidence and mortality from non-malignant diseases. Br J Cancer 1997;75:144-8.

Received for publication: 31 July 2000 